\title{
Desarrollar la historia de la metrología en Portugal: aportaciones para el estudio de las medidas de capacidad
}

Desenvolver a história da metrologia em Portugal: aportaçóes para o estudo das medidas de capacidade

To develop Metrologic history in Portugal: approaches for the study of capacity measure

\author{
Maria Alice da Silveira Tavares* \\ Universidade Nova de Lisboa, Lisboa, Portugal
}

Mário Viana. Estudos de história metrológica. Medidas de capacidade portuguesas. Lisboa: Universidade de Lisboa, Centro de História, 2015, 170p.

Estudos de história metrológica. Medidas de capacidade portuguesas es el primer volumen de Mário Viana, profesor de la Universidad de las Azores, dedicado a esta temática. Se trata de más una etapa para el desarrollo de la história económica y de la metrología, gracias a los esfuerzos del autor en aportar nuevas contribuciones e interpretaciones, en especial, a partir de fuentes portuguesas, al mismo tiempo que evalúa la bibliografía existente. Con este estudio, Viana nos ofrece una panorámica de la metrología en Portugal, desde el siglo IX hasta el XIX, proporcionándonos una perspectiva diacrónica de análisis de amplio espectro cronológico de ocho siglos. La consolidación de esta línea de estudio resulta de la sensibi- lidad y experiencia por parte del autor que se fue cristalizando a lo largo de estos últimos años, entre 2007 y 2015. Prueba de esto, son los siete capítulos que componen esta obra objeto de la presente reseña. Estos son precedidos por una breve nota previa del mismo autor con la intención de dar a conocer y de aclarar la elección de este objeto de estudio, bien como sus motivaciones. Asimismo, podemos subrayar que los dos primeros estudios tienen una función pedagógica, con los objetivos de proporcionar herramientas de trabajo (fuentes, bibliografía, por ejemplo) y de introducir al lector en las problemáticas de la historia metrológica.

El primer capítulo, "A história metrológica portuguesa. Breve roteiro ideográfico", de carácter pedagógico, tiene un doble enfoque: dar a conocer la historia metrológica portuguesa desde finales del siglo XII hasta la actualidad y hacer un punto de la situación de las investigaciones desarrolladas en este campo del conocimiento histórico,

DOI: http://dx.doi.org/10.1590/2237-101X020040012

Resenha recebida em 8 de março de 2018 e aceita para publicação em 16 de julho de 2018.

* Investigadora da Universidade Nova de Lisboa/Faculdade de Ciências Sociais e Humanas, Lisboa -

Portugal. E-mail: alice.tavares@gmail.com. http://orcid.org/0000-0001-8988-4962. 
proporcionando nuevas aportaciones. Asimismo, se pretende introducir al lector en las discusiones actuales sobre esta temática, proporcionándole algunos ejes de discusión relacionados, en primer lugar, con la idea de caos asociada a las iniciativas de uniformización del sistema métrico decimal no solo en Europa, sino también en Portugal, en perjuicio de los pesos y medidas locales. La inflación del sistema métrico decimal y a continuación, las diferencias metrológicas, según las distintas regiones de Portugal, son otros parámetros objeto de atención en este estudio. A modo de colofón, el autor nos proporciona un listado bibliográfico sobre la historia de la metrología en Portugal, proporcionándonos un instrumento de trabajo $\mathrm{y}$ un punto de orientación para futuras investigaciones en este campo del conocimiento histórico.

Dentro de la misma temática, el segundo capítulo — "Medidas de capacidade medievais portuguesas: uma revisão" — , consiste en la publicación de los resultados presentados en el evento científico ( $O$ reino, as ilhas e $o$ mar oceano. Estudos em homenagem a Artur Teodoro de Matos de Matos, edição de Avelino de Freitas de Meneses e João Paulo Oliveira e Costa, Lisboa - Ponta Delgada, Centro de História de Além-Mar - Universidade dos Açores, 2007, v. I, p. 59-80), celebrado en Portugal. Mário Viana nos hace el estado de la cuestión de las fuentes y de los estudios sobre las medidas de capacidad utilizadas durante la Edad Media en Portugal, mientras que, por otro lado, nos propone una reevaluación de la bibliografía existente sobre este objeto de estudio. Con este ejerci- cio, el autor pretende hacer una nueva reflexión sobre el sistema metrológico portugués, con el objetivo de demonstrar que se trata de un método con características propias, organizado, contradiciendo las teorías anteriores defensoras de la existencia de una métrica confusa y desordenada.

El tercer capítulo, "As medidas de capacidade nas inquiriçôes de 1258 " fue publicado anteriormente en la obra colectiva, Olhares sobre a história. Estudos oferecidos a Iria Gonçalves, Lisboa, Caleidoscópio, 2009, p. 691-702. El título nos remite directamente para el objeto de estudio - las medidas de capacidad — , observadas con base en las actas de las inquiriciones regias, fechadas de 1258, relativas a la región entre los ríos Cávado y Miño, en el Norte de Portugal. Se trata de un artículo con características peculiares, una vez que esta investigación resulta de una ósmosis entre la historia y las tecnologías, gracias a la aplicación (INQExpert) de búsqueda automatizada de texto basada en el sistema de lenguaje Java. Esta metodología posibilitó estudiar y reconstituir el sistema de medidas de capacidad tanto para sólidos, como para líquidos, en el Noroeste portugués. Este output se desarrolló en el ámbito del proyecto INQ1258, coordinado por el propio autor - Mário Viana —, que contó con financiación del Gobierno Autonómico de las Azores (Dirección Regional de Ciencia y Tecnología de las Azores, con la referencia: DRCT00276M2.1.2/I/008/2006).

Como el título nos indica — "Para a história da metrologia em Portugal: um documento de 1353 relativo a Bragança” — el presente capítulo consiste en el estudio de un 
documento relativo al concejo de Bragança (localizado en Nordeste de Portugal, cerca de la frontera con España), de la segunda mitad del siglo XIV (1353), que se encuentra en el Archivo Distrital de la referida ciudad. Este documento trata de un conflicto entre las gentes de Bragança y el concejo por culpa de las medidas de capacidad utilizadas para medir el vino. Este problema sirvió de punto de partida para explicar la política y la reforma de los pesos y medidas del rey D. Pedro I (1356-1367) que data de 1357-1358. Las informaciones de este texto fueron publicadas en la revista de la Universidad de las Azores: Arquipélago. História, Ponta Delgada, 14-15 (2010-2011). Por último, a modo de colofón, podemos apreciar la edición del documento.

El quinto capítulo - "Para a história da metrologia em Portugal: dois documentos de 1358-1360 relativos a Coimbra" —, es un ensayo con una estructura particular al igual que el anterior, pues Mário Viana trae nuevas contribuciones a partir de documentación referente a situaciones de conflictos. Una vez más, el autor se refiere a una contienda entre el concejo de Coimbra y el monasterio de Santa Cruz que asumió la defensa de la aldea de Ansiáo, término de la susodicha ciudad. Con base en los dos documentos que se encuentran editados al final del capítulo, en los anexos, el autor pretende demostrar una clara intervención gradual del poder regio en controlar las instituciones económicas locales, entre las cuales los pesos y las medidas utilizadas en los concejos y en sus términos. De este modo, se evidenció el impacto de la aplicación de la reforma metrológica otorgada en 1357-1358 por el rey
D. Pedro I (1356-1367), aunque estas conclusiones fueran publicadas, igualmente, en la revista azorina, Arquipélago. História, de la Universidad de las Azores, en el año 2009, con las páginas 281-295)

A continuación, podemos encontrar el sexto texto, "A regulação metrológica em Portugal nos séculos XV e XVI" que fue publicado, de forma parcial, en otro capítulo anterior, "A metrologia nas posturas municipais dos Açores (séculos XVI-XVIII)", en la obra colectiva: $O$ Faial e a periferia açoriana nos séculos $X V$ a XX. Actas do V Colóquio, Horta, Núcleo Cultural da Horta, 2011, p. 279-312. En este ensayo, el autor enfoca los mecanismos de reglamentación de los pesos y de las medidas y, por otro lado, reflexiona sobre los procesos de uniformización metrológica implementada por el poder regio a lo largo de la Edad Media, con el apoyo del poder local. En este sentido, el presente capítulo está organizado en dos ejes basilares: la implementación de la figura del funcionario regio, "almotacé-mor" en los concejos y su respectiva jurisdicción. El segundo dice respecto a la política de uniformización de pesos y medidas, a par de otras iniciativas que acompañan las tendencias de control del poder local y de desarrollo de una política fiscal.

En el último capítulo, "As medidas de capacidade nos Açores em 1868", consiste en un estudio que fue publicado anteriormente en la obra colectiva, Aquém e além de São Jorge: memória e visão, Lisboa, Centro de História d'Aquem e d'Além-Mar, 2014, p. 143-164. Como el título indica, Mário Viana nos adentra en las medidas de capacidad 
utilizadas en el archipiélago de las Azores, en la segunda mitad del siglo XIX, más en concreto, en 1868, en el marco de la implementación del sistema métrico decimal en Portugal. Para lograr sus objetivos, el autor se fundamenta, en especial, en una fuente decimonónica: Mapas das medidas do novo sistema legal comparadas com as antigas dos diversos concelhos do reino e ilhas. A través de esta obra, tenemos acceso a datos metrológicos de 21 "distritos" (circunscripciones administrativas) y 439 concejos portugueses. En el caso particular de las Azores, los datos observados corresponden a los "distritos” de Angra de Heroísmo (isla Terceira), Ponta Delgada (isla de São Miguel) y Horta (isla de Faial). A partir de estas indicaciones metrológicas, el autor analiza y coteja las variaciones de las medidas de capacidad para líquidos y sólidos en el archipiélago azorino, con recurso sobre todo a la cartografía isleña. En el anexo del referido capítulo, podemos encontrar una serie de tablas esquemáticas con las medidas de capacidad (tonel, pipa, almud, "pote", "canada", cuartillo, medio cuartillo y cuarto cuartillo) utilizadas en el siglo XIX, en las islas de las Azores.

En suma, se trata de una obra importante para el desarrollo de la historia económica y para el estudio de la metrología en Portugal desde la Edad Media hasta la época contemporánea. Además, Mário Viana nos aporta nuevas investigaciones, sobre todo, en consonancia con el uso de las tecnologías, herramientas de trabajo indispensables en el desarrollo de investigaciones en las ciencias sociales y humanas. 